\title{
Metamodelo de aprendizaje estratégico (MAE): Arquitectura de la capa de infraestructura, solución basada en la Cloud Computing
}

\author{
Rafaela Blanca Silva-López, Iris Iddaly Méndez-Gurrola, Oscar Herrera Alcántara \\ Universidad Autónoma Metropolitana, Distrito Federal, \\ México \\ $\{$ rbsl, ohc $\} @$ correo.azc.uam.mx, iddalym@yahoo.com.mx
}

\begin{abstract}
Resumen. En este trabajo se presenta el uso de Cloud Computing como infraestructura de un sistema de gestión del aprendizaje (LMS). El objetivo principal es que el sistema soporte la atención a cientos de alumnos bajo la modalidad de enseñanza e-Learning en una Institución de Educación Superior (IES). Algunas de las arquitecturas reportadas se especializan en otros LMS como Moodle, otras se enfocan en definir capas que integran los recursos software, hardware, los recursos de enseñanza entre otros. Existen trabajos que centran sus esfuerzos en $e$-Assessment basado en la nube. Unos más se enfocan en incluir en su arquitectura la seguridad en SOA para el control de acceso del sistema. La solución presentada en este trabajo involucra la plataforma SAKAI como LMS y la virtualización de servidores para el Cloud Computing lo cual provee una arquitectura escalable y de alta disponibilidad, y la cual representa la capa de infraestructura de un metamodelo de aprendizaje estratégico.
\end{abstract}

Palabras clave: metamodelo de aprendizaje estratégico, Cloud Computing, eLearning, e-Assessment, sistemas de gestión del aprendizaje.

\section{Introducción}

El Cloud Computing es un modelo que permite compartir un conjunto de recursos computacionales (servidores, almacenamiento, aplicaciones y servicios) en línea a través de redes. Emplea técnicas innovadoras a gran escala para acceder a recursos que se encuentran distribuidos geográficamente. Ofrece tres modalidades de servicios [1]:

a) Software como un Servicio (Software as a Service, SaaS). El proveedor ofrece aplicaciones que son utilizadas por el usuario final;

b) Infraestructura como un servicio (Infraestructure as a Service, IaaS). El proveedor proporciona infraestructura (hardware), el usuario debe instalar las herramientas que requiera para desarrollar las aplicaciones que necesita; y

c) Plataforma como un servicio (Plataform as a Service, PaaS). El proveedor ofrece utilerías sobre las que el usuario construye sus aplicaciones [3]. 
Por otro lado, se tienen cuatro modelos para implementar y ofrecer los servicios en la nube [1]:

a) Nube pública (Public cloud). El proveedor de servicios en la nube proporciona sus recursos de forma abierta a diversos usuarios;

b) Nube privada (Private Cloud). El usuario gestiona sus servicios en la nube, manteniendo el control, se centralizan los recursos informáticos lo que brinda flexibilidad en la disponibilidad de los mismos;

c) Nubes Híbridas (Hybrid Cloud). Este modelo combina servicios que se ofrecen de forma pública y de forma privada; y

d) Nube Comunitaria (Community Cloud). El proveedor ofrece los servicios que son compartidos en una comunidad cerrada.

La arquitectura del Cloud Computing integra las características y los modelos definidos previamente (ver Figura 1).



Fig.1. Arquitectura del Cloud Computing.

\subsection{Cloud Computing en las IES}

Existe una tendencia en el uso de Aplicaciones Orientadas a Servicios (SOA) [2] como mecanismos de infraestructura para la siguiente generación de Sistemas de Aprendizaje que soporten la atención a cientos o miles de alumnos bajo modalidades de enseñanza e-Learning [4]. Sin embargo, la eficiencia y rendimiento del hardware suele convertirse en el cuello de botella de estos sistemas. Esto ha provocado la necesidad de tener soluciones con una arquitectura escalable y de alta disponibilidad.

El Cómputo en la nube brinda la posibilidad de tener una arquitectura con servidores en clúster que habilitan la escalabilidad y alta disponibilidad mediante el 
uso de servidores virtualizados, además de ofrecer el software como un servicio [5]. Esta solución es una excelente alternativa viable para las Instituciones de Educación Superior como es el caso de la Universidad Autónoma Metropolitana (UAM) [6][7]. Son múltiples los beneficios que se obtienen con el uso del Cloud Computing como infraestructura de los Sistemas de Aprendizaje (LMS), los beneficios integran flexibilidad en la distribución de recursos, así como escalabilidad, almacenamiento en arreglos de discos, poder de cómputo (manejo de multiprocesadores y memoria RAM), lo que redunda en un ahorro económico [8][9].

\subsection{Tecnologías de Cloud Computing}

Las tecnologías que se encuentran atrás de una implementación de Cloud Computing son la virtualización y SOA, mismas que permiten que ésta sea flexible, confiable y extensibles. La virtualización posibilita compartir recursos físicos por demanda (memoria, storage, CPU). Al crear una máquina virtual se comparte un sistema operativo y un conjunto de aplicaciones como se muestra en la Figura 2.

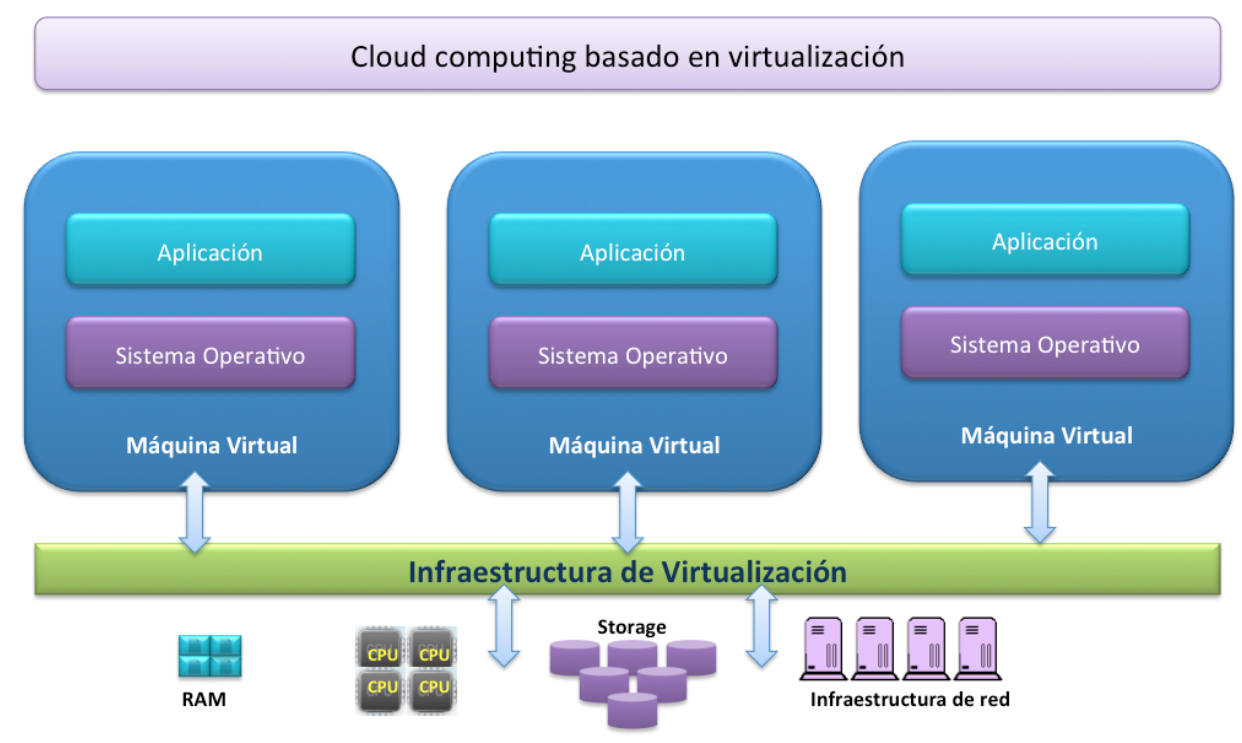

Fig. 2. Cloud Computing con virtualización.

SOA soporta el uso de aplicaciones como servicios para otras aplicaciones. Por lo tanto, es importante el intercambio de datos entre aplicaciones de diferentes proveedores sin programas adicionales.

\section{Web Services}

Un web service es una aplicación que acepta solicitudes de otros sistemas a través de Internet mediante tecnologías de comunicación independientes de la plataforma. Es 
una unidad de software autocontenida, autodescriptiva que proporcionan cierta funcionalidad a través de la infraestructura de la red Internet. El manejo de XML (eXtensible Markup Language) es central a este tipo de arquitectura, por tanto son una herramienta de integración entre plataformas heterógeneas, ya que trabaja típicamente sobre tecnologías abiertas como HTTP (Hypertext Transfer Protocol) y XML ${ }^{1}$.

\section{Service Oriented Architecture (SOA)}

SOA es un principio arquitectónico para definir sistemas distribuidos empresariales. Es un modelo para la integración de sistemas. Se han definido modelos de referencia como el administrado por OASIS2, que define un vocabulario común para ser utilizado por diferentes organizaciones, busca reducir ambigüedades $\mathrm{y}$ problemas de comunicación. Por lo tanto, se implementa utilizando tecnologías como XML, Web Sevices Description Language (WSDL) ${ }^{3}$, XML Schema $^{4}$ y SOAP 5 .

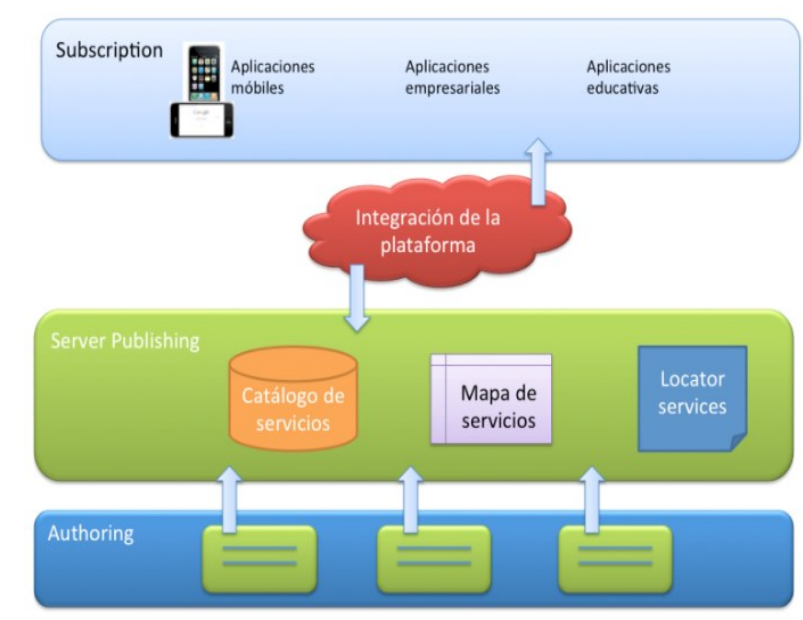

Fig. 3. Implementación de Cloud Computing con SOA.

La implementación de Cloud Computing aplicando la arquitectura SOA integra el modelo de comunicación de mensajes denominado Publish/Subscrib, en el que los subscriptores son los clientes (aplicaciones móviles, aplicaciones empresariales, entre otras), y los publicadores integran todos los servicios (catálogo de servicios) y ofrecen un mapa de servicios que se tienen disponibles desde múltiples fuentes (ver Figura 3) a través del localizador de servicios.

\footnotetext{
${ }^{1} \mathrm{http}: / /$ www.w3.org/XML/

${ }^{2} \mathrm{http}: / /$ www.oasis-open.org/committees/tc_home.php?wg_abbrev=soa-rm

${ }^{3} \mathrm{http}: / / \mathrm{www} . \mathrm{w} 3.0 \mathrm{rg} / \mathrm{TR} / \mathrm{wsdl}$

${ }^{4}$ http://www.w3.org/XML/Schema/

${ }^{5} \mathrm{http}: / /$ www.w3.org/TR/soap/
} 


\section{Arquitecturas para e-Learning basadas en la nube}

En esta sección se resumen trabajos asociados con el uso de arquitecturas basadas en Cloud Computing para soportar modalidades de enseñanza y aprendizaje mediados por Tecnologías de la Información y Comunicación (TIC). E-learning ofrece nuevas alternativas de educación mediante nuevos ambientes virtuales que facilitan la interacción y comunicación entre profesores y alumnos. El uso de la Cloud Computing en la arquitectura de Sistemas Basados en Conocimiento (SBC) que apoyan el proceso de enseñanza y aprendizaje es fundamental cuando se pretende atender una gran población de estudiantes que cursan diversos cursos que se apoyan en el uso de TIC.

\subsection{Arquitectura de un sistema e-Learning basado en la nube (India)}

La arquitectura e-Learning propuesta en[10] por los Hindúes, integra cinco capas: i) la capa de infraestructura asociada con el hardware ii) la capa de recursos de software: sistema operativo y middleware iii) la capa de recursos administrativos: servicios por demanda $i v$ ) la capa de gestión de recursos que permite el acoplamiento flexible del hardware y software, para proporcionar los servicios, y v) la capa de servicios considerando SaaS y la capa de aplicación que permite integrar los recursos de enseñanza en nube [10].

\subsection{Arquitectura de un sistema e-Assessment basado en la nube (Brasil)}

Gusev y otros [11], resumen las cuatro generaciones de sistemas e-Learning y $e$ Assessment. La primera generación considera métodos monolíticos basados en tecnologías stand-alone; la segunda generación se basa en métodos modulares y tecnologías web; para la tercer generación se aplican métodos orientados a servicios en los que se involucran tecnologías hypermedia adaptativa y web semántica; finalmente, la cuarta generación usa métodos de cómputo en la nube, soportado por clústers de servidores y la nube. Proponen una arquitectura de $e$-Assessment basada en la nube, como una solución sustentable de bajo costo. La arquitectura está constituida por tres módulos: administración, e-Assessment y reports. El módulo de administración es el núcleo del sistema e-Assessment, está constituido por la capa de infraestructura que gestiona el hardware y la capa de servicios que provee los servicios en la nube (Learning Management System, Virtual Learning Enviroment y Management Information System) [11].

\subsection{Smart e-Learning usando Cómputo en la nube y Web 3.0 (Egipto)}

Pjanic de la University of Tuzla, Tuzla, Bosnia and Herzegovina, su arquitectura se basa en la solución de cómputo en la nube proporcionada por Microsoft Windows Azure platform y web 3.0, siendo una solución de bajo costo aplicable en Instituciones Educativas [12]. Los componentes de la arquitectura que proponen 
incorporar son: a) servicios basados en web (aprendizaje y evaluación); b) modelo basado en una base de conocimiento; y c) el cómputo en la nube utilizando Windows Azure. Los beneficios que se reportan de integrar el cómputo en la nube y la web 3.0 son calidad en el servicio, escalabilidad, uso eficiente de recursos, sustentabilidad, estabilidad, entre otras.

\subsection{U-SEA: e-Learning basado en la nube (Brasil)}

U-SEA es una propuesta de Piovesan y otros de la Universidad Federal de Santa María (UFSM) de Brasil. Su propuesta se fundamenta en Moodle $^{6}$ y un módulo desarrollado para la adaptación del entorno del estudiante a través de dispositivos móviles, soportado por tecnología de cómputo en la nube. La arquitectura es muy simple, integra como LMS a Moodle y el módulo adaptativo que accede una base de datos que contiene la información para determinar los mecanismos de adaptación del contexto. Mientras que la infraestructura considera el mecanismo de cómputo en la nube privado, y el modelo de SaaS [13].

\section{5. "Cloud Education" (Egipto)}

La arquitectura de "Cloud Education" basada en la nube, se constituye a partir de una capa física en la que incluyen el hardware necesario (almacenamiento, servidores, red), una capa de virtualización (servidores, almacenamiento y base de datos virtuales), la capa de middleware educación (plataforma educative, administrador de profesores, recursos educativos), una capa de aplicación (gestión de usuarios, recursos y cursos). A diferencia de las anteriores arquitecturas considera una arquitectura de seguridad basada en SOA para el control de acceso [14].

\subsection{E-Learning en la nube, basado en la especificación IMS-LD. University of Hassan}

El modelo propuesto por denominado Instructional Management SystemsLearning Design (IMS-LD), adopta el uso de contenidos, habilita la interoperabilidad, facilita la adaptación de recursos para la enseñanza y el aprendizaje a través de la nube. En su propuesta integran un servicio pedagógico y en su implementación integran el e-Learning y el cómputo en la nube. También contemplan un proceso de diseño instruccional [15].

La arquitectura en la nube para la Universidad contempla un modelo de desarrollo (Nubes públicas, privadas, híbridas y comunitarias), un modelo de servicios (SaaS, PaaS, IaaS) y recursos móviles (e-Learning, archivo digital, portales, servicios de hosting). Para el caso que presentan, utilizaron Nube comunitaria y PaaS [3].

\footnotetext{
${ }^{6} \mathrm{https}: / /$ moodle.org/?lang=es 


\section{Arquitectura de la capa de infraestructura como una solución basada en la nube}

En esta sección se propone la arquitectura y organización de la capa de infraestructura del Metamodelo de Aprendizaje Estratégico (MAE) [16].

El MAE integra los principios de la evaluación mediadora, la personalización de las actividades de aprendizaje, la supervisión, seguimiento y atención personalizada, así como el trabajo de las comunidades de aprendizaje con el objetivo de brindar el fortalecimiento de habilidades para el trabajo en equipo, el liderazgo y la negociación, entre otros. El MAE optimiza los recursos físicos y humanos de una institución, buscando disminuir los niveles de deserción e incrementar los niveles de aprobación. En la Figura 4 se muestra el metamodelo, en donde se aprecian las capas de infraestructura, inteligente y reactiva.

La solución busca optimizar los costos y los recursos existentes para atender grupos de 50 a 250 alumnos de ingeniería.

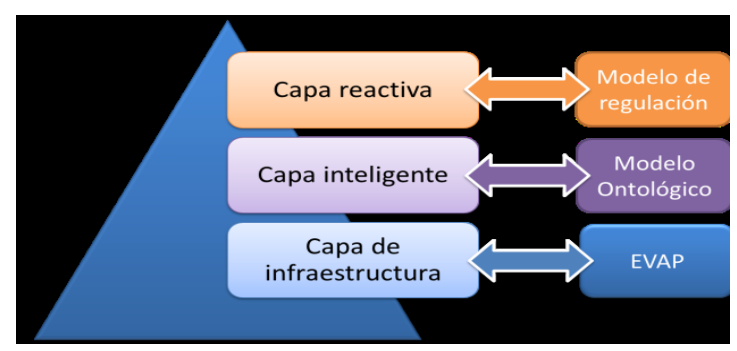

Fig. 4. Modelo de Aprendizaje Estratégico (MAE).

\subsection{Arquitectura de la capa de infraestructura del MAE}

La arquitectura de la solución de Cloud Computing para la capa de infraestructura del MAE, integra cuatro componentes: Learning Mangement System (LMS), Personalized Virtual Learning Enviroment (PVLE), Management Information System (MIS) que maneja la información del Ontological Model (OM). Los componentes se implementan como una solución del tipo Cloud Computing, tomando como base el trabajo de Christian Gütl (2008) y Gusev, Ristov, Amenski, Velkoski y Bozinoski [11]. Nuestra propuesta integra además el modelo ontológico para la personalización de actividades de aprendizaje que reemplaza el módulo MIS que propone Gusev, como se muestra en la Figura 5.

\subsection{Learning Management System (LMS)}

Se establece como plataforma LMS a $\mathrm{SAKAI}^{7}$, se aprovechan diversas herramientas que se integran como el chat, los foros, los avisos, y se incorpora el uso

${ }^{7}$ https://sakaiproject.org/ 
de videoconferencia apoyándose en el software de Adobe Open Connect ${ }^{8}$. Adicionalmente se aprovechó el uso de las redes sociales y se determinó la creación de un espacio en Facebook para mantener contacto permanente con los alumnos, como se muestra en la Figura 6.

El LMS utilizado es SAKAI, desarrollado en lenguaje Java lo que permite integrar otros desarrollos en el mismo lenguaje, es portable y se puede instalar en múltiples plataformas.

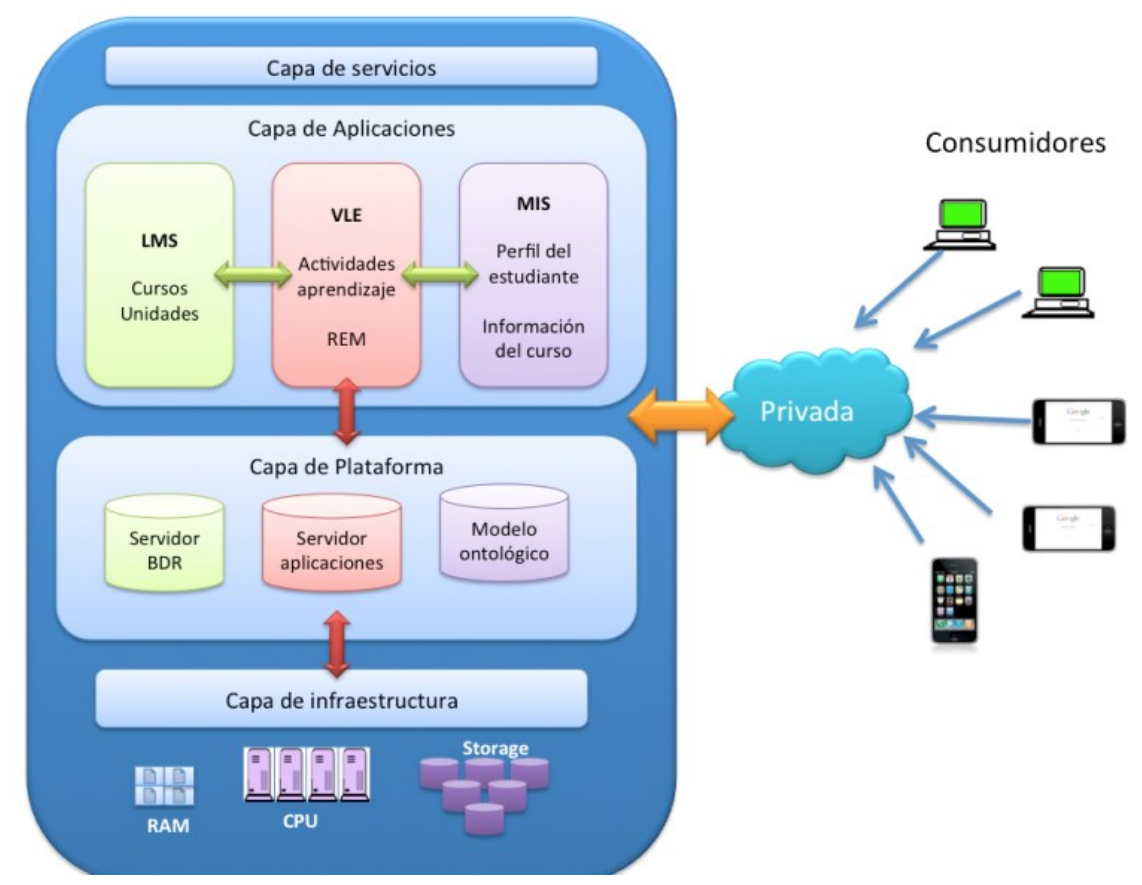

Fig. 5. Arquitectura de integración del Modelo en la Cloud Computing.

La capa de datos de SAKAI está desarrollada con el framework Hibernate ${ }^{9}$ que permite realizar un mapeo Objeto-Relación (OR) entre los objetos de Java y los campos de las tablas de la base de datos. El mapeo OR habilita la portabilidad para el uso de múltiples manejadores de bases de datos con el simple hecho de cambiar el driver correspondiente. En la Figura 6 se muestra la arquitectura del LMS SAKAI. La plataforma actualmente soporta hasta 8,000 usuarios (600 concurrentes), debido a los recursos de hardware asignados. La propuesta es escalable en el futuro si aumentara la carga mediante la asignación de más recursos de procesamiento, memoria y almacenamiento mediante la infraestructura de virtualización.

\footnotetext{
${ }^{8} \mathrm{http}: / / \mathrm{www}$.adobe.com/mx/products/adobeconnect/apps.html

${ }^{9} \mathrm{http}: / /$ hibernate.org/ogm/
} 


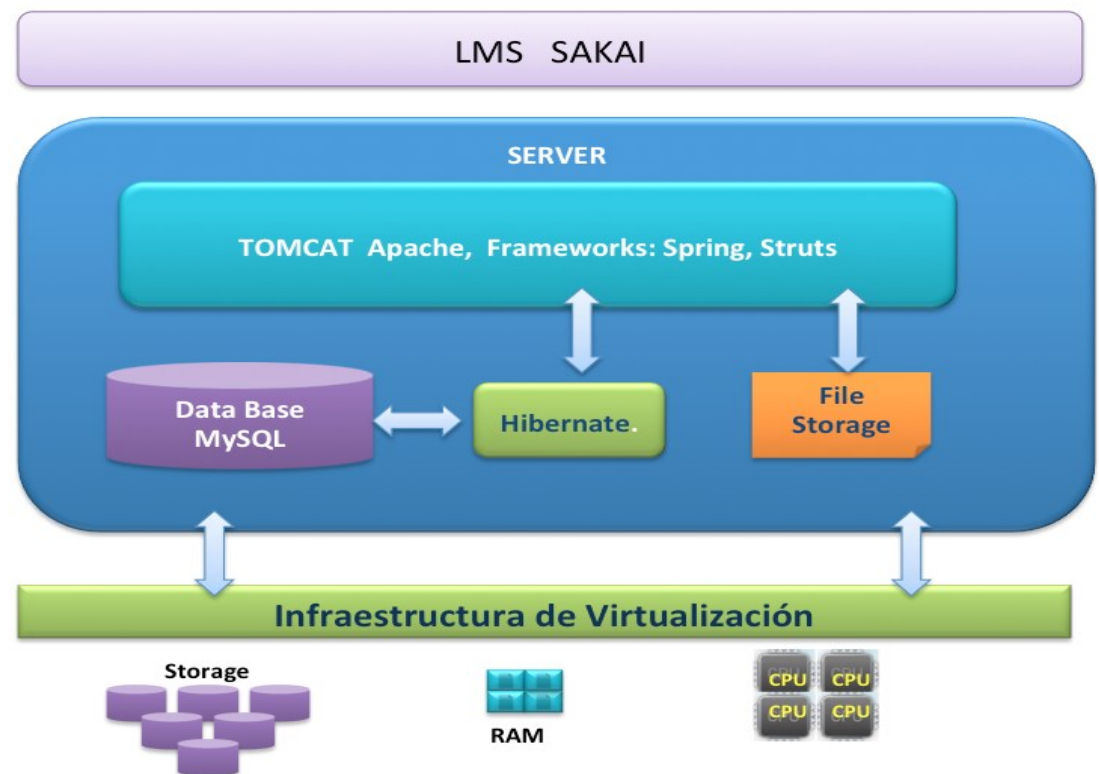

Fig. 6. Arquitectura de soporte para el LMS SAKAI.

\subsection{Entorno virtual de aprendizaje personalizado y modelo ontológico}

Para llevar a cabo el desarrollo, implementación y pruebas, del Entorno Virtual de Aprendizaje Personalizado (EVAP), es conveniente definir los componentes que se usará como fundamento tecnológico.

El EVAP es un entorno adecuado para el aprendizaje, ya que en él se integran múltiples herramientas que tradicionalmente se usan en Internet tal como el uso de redes sociales, así como recursos educativos multimedia. Para tal fin se elaboraron diversos recursos educativos multimedia, así que el alumno tiene la libertad de usar el recurso que desee. Inclusive, si el tema no le queda claro a partir de un recurso, puede acceder los recursos de otro estilo y complementar su aprendizaje; al mismo tiempo que desarrolla sus otros canales de percepción y habilidades como receptor crítico. Adicionalmente se incorpora el uso y creación de mapas mentales como herramienta para integrar el conocimiento.

El EVAP se propone como un sistema en el que se integrará el Modelo Ontológico (MO) como parte del trabajo futuro. El EVAP se constituye por un servidor de aplicaciones, tres frameworks: uno para la centralización de servicios $\left(\right.$ Spring $\left.{ }^{10}\right)$, otro para el mapeo a base de datos (Hibernate), y un tercero para la implementación del controlador y vista $\left(\right.$ Struts $\left.^{11}\right)$. Considera la conexión a ontologías que conforman el modelo ontológico para la personalización de actividades de aprendizaje como se muestra en la Figura 7.

\footnotetext{
${ }^{10} \mathrm{http}: / /$ projects.spring.io/spring-framework/

${ }^{11} \mathrm{https} / / /$ struts.apache.org/
} 


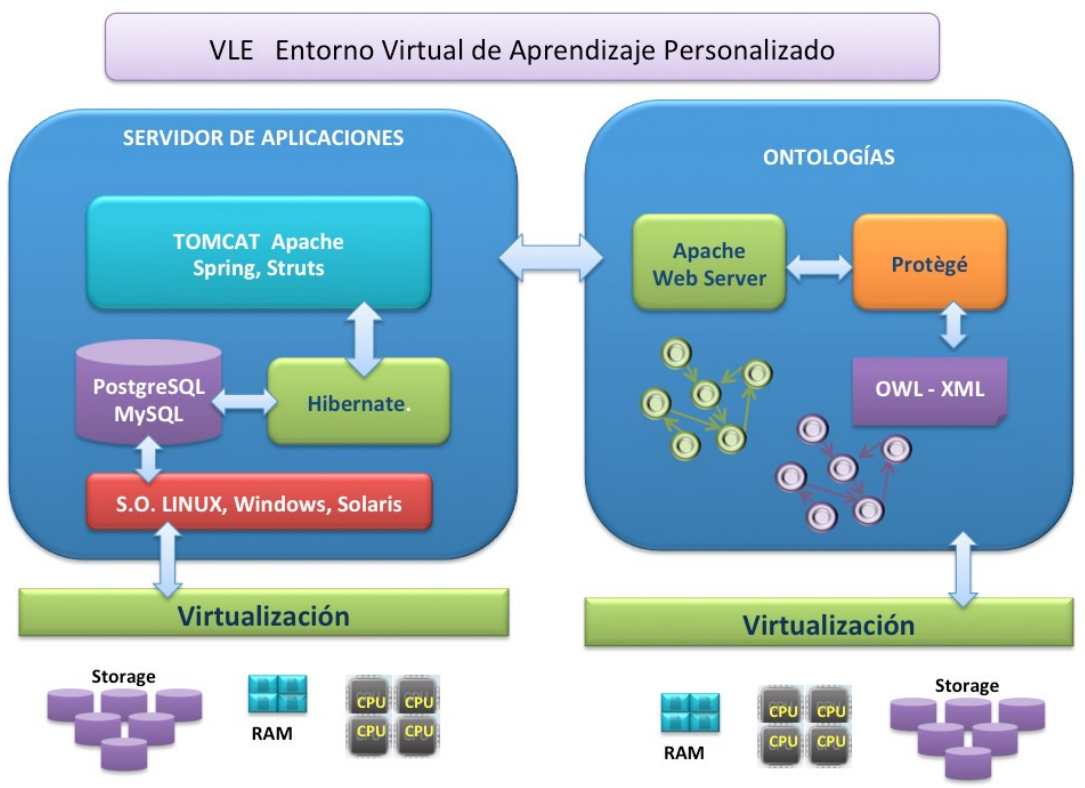

Fig. 7. Arquitectura del EVAP y Modelo ontológico.

\subsection{Infraestructura tecnológica}

Teniendo en cuenta las bondades que ofrecen las soluciones de Cloud Computing se presenta una arquitectura tecnológica que integra los componentes de la solución en la que se incluye el modelo ontológico propuesto en este trabajo de investigación.

$\mathrm{La}$ arquitectura utiliza como modalidades de servicios Plataform as a Service (PaaS) [3], ya que proporciona el acceso a manejadores de bases de datos $\left(M y S Q L^{12}\right.$, PostgreSQL $L^{13}$ ), servidores de aplicaciones (Apache TOMCAT ${ }^{14}$ ), entornos de programación $\left(\right.$ Eclipse $^{15}$, NetBeans $\left.^{16}\right)$, compiladores $\left(C, C++\right.$, Java $^{17}, \mathrm{PHP}^{18}$, entre otros), y sistemas operativos (Linux ${ }^{19}$, Solaris ${ }^{20}$, Windows $\left.^{21}\right)$, sobre las que el usuario construye las aplicaciones que necesita. Los productos y marcas son propiedad de las respetivas compañías.

Como modelo de implementación para ofrecer los servicios en la nube se utiliza tanto la Private Cloud (Nube privada) como la Community Cloud (Nube

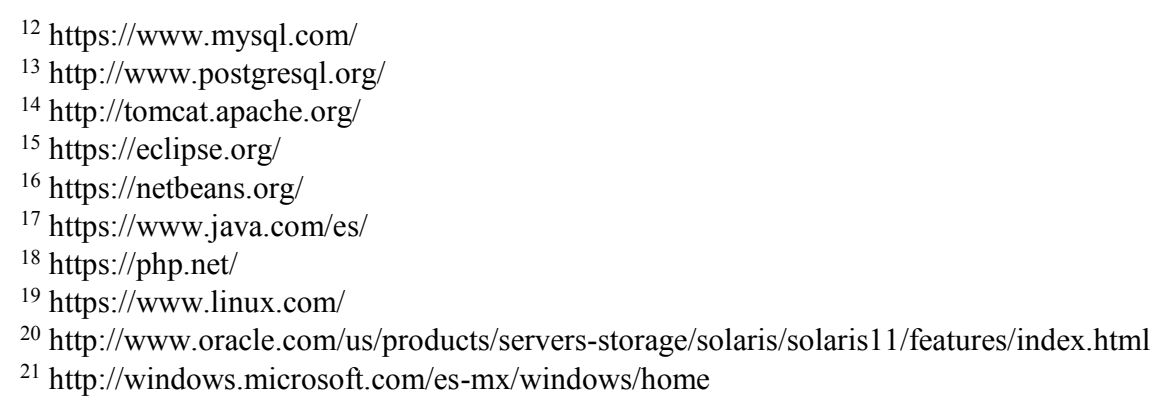


Comunitaria). Los servicios son compartidos en una comunidad cerrada al interior de la Universidad.

Por último, la tecnología base para la implementación de Cloud Computing es la virtualización, ya que ofrece disponibilidad, flexibilidad, confiabilidad y extensibilidad. Los recursos de espacio en disco, memoria y CPU se distribuyen dinámicamente de acuerdo con la demanda en las máquinas virtualizadas disponibles, como se muestra en la Figura 7.

La infraestructura tecnológica con la que cuenta la Institución de Educación Superior incluye 4 servidores Blade HP Proliant BL 465c G7, cada uno con 24 microprocesadores AMD Opteron 6174 de $2.199 \mathrm{GHz}$, con licencia VMware vSphere 5 Desktop y 16 GB de memoria RAM configurada por servidor, ver Figuras 8 y 9.

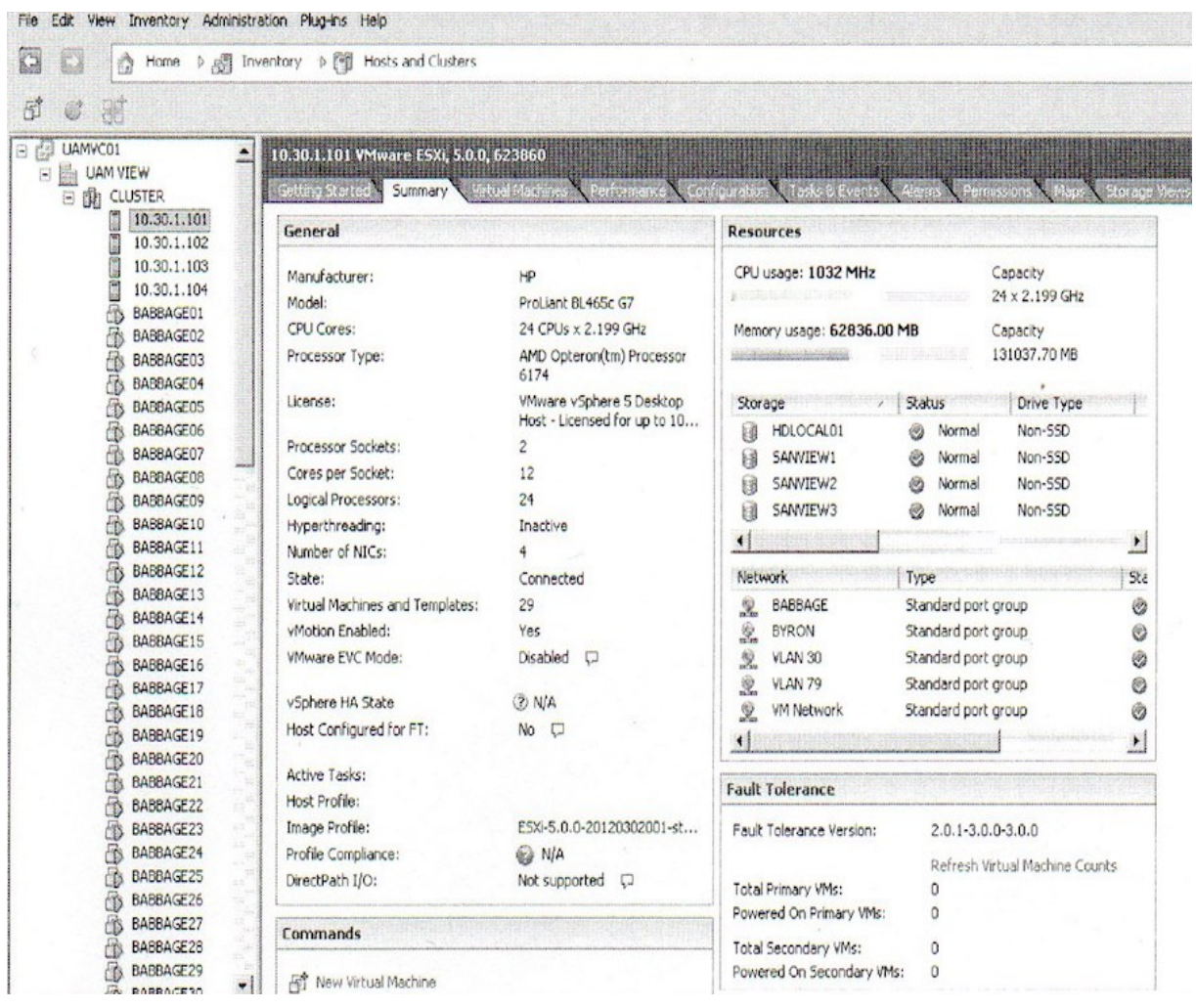

Fig. 8. Servidores Blade HP Proliant BL 465c G7, con VMware vSphere 5.

Se utiliza el software VMware vSphere 5 para llevar a cabo la virtualización de los recursos que se distribuyen de manera dinámica con el objetivo de optimizar los recursos existentes.

En cuanto a la seguridad, se aplica el aislamiento con el hipervisor incorporado, detección de intrusiones, antivirus, escaneo de vulnerabilidad. Se aplican los mecanismos de seguridad específicos de las VM incrustadas en APIs del hipervisor 
utilizadas para proporcionar seguimiento granular del tráfico. Se tiene un política de respaldos y restauración que contempla la gestión de cambio y configuración, considerando la gestión de parches, mantenimentos programados trimestralmente.



Fig. 9. Cluster de Servidores Blade HP Proliant BL 465c G7, con VMware vSphere 5.

\section{Conclusiones}

En este trabajo, se describe la arquitectura de la capa de infraestructura del metamodelo de aprendizaje estratégico (MAE), implementado como una solución en la nube. Uno de los puntos importantes de nuestra arquitectura a diferencia de las presentadas en la sección 2, es la incorporación de las arquitecturas de los cuatro componentes del MAE: LMS, el EVAP, el Management Information System (MIS) y el MO. La implementación en la nube se realiza a partir de servidores virtualizados que utilizan recursos físicos de hardware sobre demanda. El modelo de implementación utilizado es tanto Private Cloud, como Community Cloud ya que los servicios se comparten al interior del Campus de la Institución de Educación Superior. En cuanto a la modalidad del servicio se utiliza PaaS para tener acceso a servicios como el manejador de la base de datos, los servidores de aplicaciones y los entornos de programación. La seguridad es revisada a través del monitoreo de las aplicaciones y las bases de datos que se integran en el modelo propuesto. Dado que se implementó un esquema PaaS, en una Cloud Private, los Sistemas Operativos virtualizados incluyen controles de seguridad por capas: a nivel aplicación (con controles de 
acceso), a nivel base de datos, a nivel red (firewall). Se crearon zonas de seguridad y se dividieron los ambientes de desarrollo y producción.

Como trabajo futuro se espera integrar ontologías asociadas con los estilos de aprendizaje y pensamiento que faciliten a su vez la integración de técnicas de inteligencia artificial para personalizar la enseñanza y el aprendizaje del alumno.

\section{Referencias}

1. Machado, J. C.; Moreira, L. O., Sousa, F. R. C.: Computação em Nuvem: Conceitos, Tecnologias, Aplicações e Desafios. In: ERCEMAPI (2010)

2. Blinco, K., Mason, J., McLean, N., Wilson, S.: Trends and issues in e-learning infrastructure development. Altilab04, Redwood City, California, USA (2004)

3. Jansen, W. A.: Cloud Hooks: Security and Privacy Issues in Cloud Computing NIST Proceedings of the 44th Hawaii International Conference on System Sciences (2011)

4. Dagger, D., O'Connor, A., Lawless, S., Walsh, E., Wade, V.: Service oriented e-learning platforms: From monolithic systems to flexible services. Internet Computing, IEEE, vol. 11, no. 3, pp. 28-35, http://dx.doi.org/10.1109/MIC.2007.70 (2007)

5. Armbrust, M., Fox, A., Griffith, R., Joseph, A., Katz, R., Konwinski, A., Lee, G., Patterson, D., Rabkin, A., Stoica, I. et al.: A view of cloud computing. Communications of the ACM, vol. 53, no. 4, pp. 50-58, http://dx.doi.org/10.1145/1721654.1721672 (2010)

6. Mircea, M., Andreescu, A.: Using cloud computing in higher education: A strategy to improve agility in the current financial crisis. Communications of the IBIMA, vol. 2011 (2011)

7. Chandra, D., Borah, M.: Cost benefit analysis of cloud computing in education. In: Computing, Communication and Applications (ICCCA), 2012 International Conference on, IEEE, pp. 1-6 (2012)

8. Ghazizadeh, A.: Cloud computing benefits and architecture in elearning. In: Wireless, Mobile and Ubiquitous Technology in Education (WMUTE), 2012 IEEE Seventh International Conference on, pp. 199-201 (2012)

9. Fernández, A., Peralta, D., Herrera, F., Benitez, J.: An overview of e-learning in cloud computing. In: W. on Learning Techn. for Educ. in Cloud (LTEC'12), ser. Advances in Intelligent Sys. And Comp., L. Uden et al., Ed., Springer, vol. 173, pp. 35-46 (2012)

10. Vasanthi, M., Magboul, I., Anandhavalli, M.: Moving towards Virtual Learning Clouds from Traditional Learning. Higher Educational Systems in India, Vol. 9, No. 9, Special Issue "Blended Learning" (2014)

11. Gusev, M., Ristov, S., Armenski, G., Velkoski, G., Bozinoski, K.: E-Assessment Cloud Solution: Architecture, Organization and Cost Model. Ss. Cyril and Methodius University, Skopje, Macedonia, Volume 8, Issue 2, http://dx.doi.org/10.3991/ijet.v8iS2.2783 (2013)

12. Nasr, M., Ouf, S.: A Proposed Smart E-Learning System Using Cloud Computing Services. In: PAAS, IAAS and Web 3.0, Helwan University, Helwan, Egypt; iJET, Volume 7, Issue 3, http://dx.doi.org/10.3991/ijet.v7i3.2066 (2012)

13. Piovesan S., Amaral E., Arenhardt C., and Medina R.: U-SEA: A Learning Environment Ubiquitous Using Cloud Computing. Universidade Federal de Santa Maria - UFSM, Santa Maria, Brazil; iJET, Volume 7, Issue 1, http://dx.doi.org/10.3991/ijet.v7i1.1838 (2012)

14. Hosam, F., El-Sofany, A. A., Khalid, A., Samir, A.: The Impact of Cloud Computing Technologies in E-learning. British University in Egypt (BUE), Cairo, Egypt; iJET, Volume 8, Issue 1, http://dx.doi.org/10.3991/ijet.v8iS1.2344 (2013) 
Rafaela Blanca Silva-López, Iris Iddaly Méndez-Gurrola, Oscar Herrera Alcántara

15. Ouahabi, S., Eddaoui, A., Labriji, E.H., Benlahmar, E., Guemmat, K.: Implementation of a Novel Educational Modeling Approach for Cloud Computing. University of Hassan II, Mohammedia, Casablanca, Morocco; iJET, Volume 9, Issue 6, http://dx.doi.org/10.3991/ijet.v9i6.4153 (2014)

16. Silva-López, R., Méndez-Gurrola, I., Sánchez-Arias, V.: Strategic learning, towards a teaching reengineering. Research in Computing Science, vol. 65, pp. 133-145 (2013) 